\title{
Confrontation Naming and Generative Naming in Dementia: A Comparison
}

\section{Deepthi Vivek $\mathrm{V}^{1 *}$ and Amirtha Varshini $\mathrm{MJ}^{2}$}

${ }^{1}$ Audiologist and Speech Language Pathologist, Private Practice, India

${ }^{2}$ Professor, Department of Speech and Hearing, MERF-Institute of Speech and

Hearing, India

*Corresponding Author: Deepthi Vivek V, Audiologist and Speech Language

Pathologist, Private Practice, India.
Received: July 20, 2021

Published: August 16, 2021

(C) All rights are reserved by Deepthi Vivek V

and Amirtha Varshini MJ.

\section{Abstract}

The Picture naming test is a widely accepted method to assess the cognitive deterioration in patients with Dementia. It is presumed to require access to semantic memory and so would be a valuable tool in analysing the nature of semantic deterioration. Language functioning and social performance has been to gradually decrease with progression of the disease. The aim of this study was to identify the differences between confrontation naming and generative or divergent naming in patients with dementia of Alzheimer's type and to provide insight into the representations within semantic memory. Both of these naming tasks have been found to be effective in identifying dementia as well as provide insight into the progression of the disease. Confrontation naming involves direct selection of a semantic memory category in response to a visually presented stimulus. Divergent naming includes accessing a multitude of words that are triggered by the presentation of auditory stimuli. The confrontation naming task used was picture naming and the generative or divergent naming task was verbal fluency task. The current study considered a control group (30) and an experimental group with age and gender matched individuals (30). The picture naming task consisted of 20 pictures of commonly used everyday objects. Category Fluency Task with Subtask I (living) and subtask II (non-living) was used. There was a noticeable difference in performance in both tasks between the control group and experimental group. It is also important to note that the experimental group performed significantly lower in the divergent naming task compared to the confrontational naming task. This might be attributed to the presence of the visual stimuli contributing as a cue in the confrontation naming task. Divergent naming or Category fluency necessitates the recall of multiple responses from a hierarchically and categorically organised information. This indicates that naming tasks can be used to identify semantic deterioration, thereby aiding in the early diagnosis and intervention of individuals with Dementia.

Keywords: Dementia; Naming; Semantic Memory; Confrontation naming; Divergent Naming

\section{Abbreviations}

LTM-Long Term Memory; STM- Short Term Memory

\section{Introduction}

Memory is an important aspect of human cognition and refers to the storage and retrieval of any and all forms of information.
The three major classifications of memory are as follows: sensory memory, short-term memory (STM), and long-term memory (LTM). Information from our environment is stored by sensory memory, making it possible for this information to be accessible in the future. STM refers to the information processed by a person in 
a short period of time. Working memory performs this processing. LTM allows us to store that information for long periods of time. This information may be retrieved consciously (explicit memory) or unconsciously (implicit memory) [1].

In long-term memory, there is declarative and non-declarative memory. Within declarative memory, we find both episodic memory and semantic memory.

Episodic memory is the concept of recollecting instances that happened in one's own past. It is different from Semantic memory in the sense that semantic memory is factual and context based.

Human beings can represent concepts in language. This ability allows us not only to communicate conceptual knowledge to others, but also to manipulate, associate, and combine these concepts. Binder and Desai found that "humans use conceptual knowledge for much more than mere interaction. Everything about humans, culture, science, literature, social institutions, religion, and art, is constructed from conceptual knowledge" [2]. Reasoning, planning for the future or reminiscing about the past depend on this conceptual knowledge which is stored in semantic memory [3].

The specific sensory, motor and emotional modality all participate in understanding language and there are large regions of the brain (the inferior parietal lobe and a large part of the temporal lobe) involved in tasks related to understanding. All these regions are involved in perception processing, and their united involvement allow supramodal representations of perceptual experience. Conceptual functions, including language, social cognition, object recognition, and the extraordinary human ability to remember the past and imagine the future all stem from this supramodal representation [2]. Consequently, it is agreed that semantic memory consists of two representations: a specific modality and supramodal modality.

LTM refers to unlimited information storage that can be maintained for long periods, even for life.

Implicit memory includes all unconscious memories, as well as certain abilities or skills. The four types of implicit memory are procedural, associative, non-associative and priming.

\section{Semantic memory}

Semantic memory is an organised knowledge of words and necessary for the use of language, it contains information and knowl- edge of the world, of objects, facts, concepts, features and words and their meanings $[6,7]$.

Episodic and Semantic memory are two information processing systems that (a) selectively receive information from perceptual systems or other cognitive systems, (b) retain various aspects of this information and (c) upon instruction, transfer specific retained information to other systems, including those responsible for translating it into behaviour and conscious awareness. The two systems are different from each other in the sense of the nature of stored information, the autobiographical versus cognitive reference, the conditions and consequences of retrieval and also in terms of their dependence upon each other.

Episodic memory perceives and stores information about temporally dated episodes or events, and temporal-spatial relations between these events. A perceptual event can be stored in the episodic system completely as to its perceptible properties or attributes and it is always stored in terms of its autobiographical reference to the already existing contents of the episodic memory store, also making the retrieved contents accessible to inspection. The system is quite susceptible to modification and/or loss of information. The form of external information or input registered in the episodic memory can be strongly influenced by the semantic memory store. This phenomenon is known as encoding- it is also possible for the episodic system to operate relatively independently of the semantic system.

Semantic memory is extremely important for language, it is a mental thesaurus. It is the organised knowledge a person possesses about words and other verbal symbols, their meaning and reference, about relations among them, and about rules, formulas, and algorithms for the usage of these symbols, concepts and relations. Semantic memory does not register the perceptual property of inputs, but rather cognitive referents of input signals. The semantic system permits the retrieval of information that was not directly stored in it, and retrieval of information from the system leaves its contents unchanged, although any act of retrieval constitutes an input into episodic memory. The semantic system is additionally much less susceptible to involuntary transformation and loss of information than the episodic system. Finally, the semantic system maybe quite independent of the episodic system in recording and maintaining information since identical storage consequence maybe brought about by a great variety of input signals. [6]. Fur- 
thermore, semantic memory is presumed not to be localized to one neuroanatomical region but rather to reflect activation of modalityspecific information encoded within a widely distributed network of regions. This hypothesis is supported by various articles carried out on functional imaging of semantic processing, on normal individuals as well as individuals with neuropsychological disorders [8].

Dementia is a condition characterized by a decline in cognitive function and memory from previously attained intellectual levels, which is sustained over a period of months or years. The deterioration is of such severity that it impairs the affected individual's ability to work, and to perform activities of daily living, including communication [9]. There are a number of types of dementia, from Dementia of Alzheimer,s type to vascular dementia, lewy body dementia and Fronto temporal dementia. Though each type of dementia is different, it has overlapping symptoms and concurrent comorbidities. Alzheimer's disease (AD) is characterized by prominent episodic memory impairment whereas in other types of dementia, other domains are more primarily affected, such as executive functioning in vascular dementia (VaD) [10]. Contrary to Alzheimer's disease, patients with semantic dementia have relatively intact episodic memory as seen by normal orientation and good memory for recent life events. They perform poorly on tests of anterograde verbal memory, but show normal, or near normal, performance on visually based recognition memory tests until late in the course of the disease [11].

Though there are multiple studies that discuss the semantic memory components between patients with different types of dementia, very few studies compare the efficiency of one task of semantic memory over the other. This study would give information on the efficacy of the tests used and to the extent they contribute to the assessment of patients with Dementia of Alzheimer's type.

\section{Aim of the Study}

The aim of this study was to compare the performance of patients with Dementia of Alzheimer's type and a control group of age and gender matched individuals on tasks of Semantic memory. The tasks used were picture naming and Category fluency.

\section{Methodology}

In order to compare and investigate the relationship between confrontation naming and generative naming, two tasks were used.
The first task was picture naming and the next, Category fluency.

The study consisted of two groups, Group I and Group II. Group I was the experimental group and consisted of 30 adults between the ages of 55 - 80 years, diagnosed with Dementia of Alzheimer's type. They were all assessed by neuropsychological measures and found to have cognitive deterioration. All individuals were chosen based on their Mild to moderate range of difficulty in both MMSE (Mini Mental Status Examination) and CDRS (Clinical Dementia Rating Scale).

Group II was the control group made of 30 age and gender matched normal aging adults. These subjects were found to have scores in the normal level in both MMSE and CDRS. Persons with other neurological deficits or interfering issues such as stroke, alcoholism, drug abuse, learning disability, head injury or major medical illnesses were excluded.

\section{Task I: Naming}

To assess the picture naming ability, 'naming', one of the subtests of Western Aphasia Battery (WAB), developed and standardized by Andrew Kertesz in 1982 [12] was selected. In WAB, it is a object naming task. For this purpose, it was modified into a picture naming task with 20 pictures of common objects used in everyday situations. All pictures were in colour.

\section{Instructions}

The subjects were instructed to name the picture that they are presented with, before showing pictures of the target items. Demonstration was carried out before the actual task. In the absence of an immediate response, the client was provided with a phonemic cue to elicit the response. The reaction time for each item was recorded.

Scoring

The responses were scored based on the scoring that was given in the WAB test tool. If there was no or incorrect responses to the visual stimulus, a phonemic cue was presented. A maximum of 20 seconds was allowed for each item. A score of 3 was given if named correctly, 2 for a recognizable phonemic paraphasia, and 1, if a phonemic cue was required. A maximum score of 60 is possible in this task 
Category fluency task (CFT) or divergent naming task

Category fluency tasks are a measure of executive functions and language. It had two subtasks. Subtask I was Living Things in which the subjects were asked to produce as many exemplars as possible from the 'animal' category and Subtask II was man made objects in which the subjects were asked to produce as many exemplars as possible from the 'supermarket items' category. For each category, the subjects were allowed 60 seconds to generate as many words as possible orally.

\section{Instructions}

Subjects were instructed as follows: "I'm going to give you a category and ask you to name all the different examples that you can think of from that category in one minute. For instance, if I said vegetables, you might say carrot, tomato etc".

\section{Scoring}

The scoring was given based on the number of items correctly produced within each category. A score of 1 was given for every correct response. Repetitions were not considered for scoring. A mean score of 12.58 - 18.51 is considered to be normal for South Indian adults [13].

\section{Results and Discussion}

The scores obtained in this method was analyzed using Statistical package for social sciences (SPSS) software (17.0 version). Independent $t$ test was used to compare the significant difference between groups and gender for each task. The ' $p$ ' value of significance thus obtained was considered to be $\geq 0.05$. the performance score for each subtest was described using the statistical mean and standard deviation.

\begin{tabular}{|l|c|c|c|c|c|c|}
\hline & $\begin{array}{c}\text { Number of } \\
\text { subjects }\end{array}$ & $\begin{array}{c}\text { Maximum } \\
\text { score }\end{array}$ & Mean & $\begin{array}{c}\text { Standard } \\
\text { Deviation }\end{array}$ & $\begin{array}{c}\text { LD evel of } \\
\text { significance }\end{array}$ \\
\hline Group I & 30 & 60 & 38.80 & 9.725 & 1.776 & 0.000 \\
\hline Group II & 30 & 60 & 54.73 & 4.143 & 0.756 & 0.000 \\
\hline $\begin{array}{l}\text { (p value } \leq 0.05 \\
\text { indicates significant } \\
\text { level of difference) }\end{array}$ & & & & & & \\
\hline
\end{tabular}

Table 1

In the naming task, Group I obtained a mean score of 38.80 which is lowest compared to Group II, that obtained a mean of 54.73. The Standard Deviation values were 9.725 and 4.143 for Group I and Group II respectively. Statistical analysis through Independent sample t test revealed that the scores of Group I and Group II were statistically significant with a " $\mathrm{p}$ " value of 0.000 (p $\leq 0.05)$.

These results are in line with the reports of Hodges., et al. in 1996 [41] who studied the relationship between naming and the integrity of physical and associative knowledge in patients with DAT and matched controls. They concluded that DAT subjects performed significantly lower in naming tasks compared to that of matched controls supporting the view that object naming depends upon access to a specific subset of semantic knowledge.

Silagi., et al. in 2015 [15] also found that Verbal semantic paraphasia were the most frequent errors found in patients with $\mathrm{Al}$ - zheimer's disease. As the disease severity increased, the number of no-response errors increased. They also found that the group of individuals with moderate Alzheimer's disease demonstrated a greater incidence of visual errors and unrelated verbal paraphasias compared with the other two groups and exhibited with a variety of errors.

Naming deficits is an indicator of overall language and cognitive dysfunction and can be used to assess the importance of both perceptual and linguistic factors. Perceptual difficulty and word frequency contributed significantly to naming performance compared to word length, were in demented patients [16].

Greater number of lexical errors observed in DAT subjects (Group I) for naming task is also been reported by Weiner, Neubecker, Bret and Hyman in 2008 [17]. While exploring the confrontational naming ability of Alzheimer's disease patients, they 
reported that relative to control subjects, $\mathrm{AD}$ patients committed more number of lexical errors when asked to name a target object.

Impaired picture naming by Group I was characterized by patients choosing words semantically related to the correct names of objects. For example, for fork, the frequent error observed was / karandi/, i.e. ladle. Similarly, for pipe the response obtained was rod. Huff., et al. in 1986 [40] reported similar findings in Alzheimer's disease patients using visually presented object naming task. They concluded that patients with AD have a semantic impairment that leads to difficulty in distinguishing among objects that are members of the same semantic category, and that this in turn leads to difficulty producing the names for objects.

Out of the 20 items, the poorest mean was obtained for the $8^{\text {th }}$ item (eraser) for both the groups. Other items performed poorest were $13^{\text {th }}$ (paperclip), $14^{\text {th }}$ (pipe) and $19^{\text {th }}$ (fork) by Group II. Though the above mentioned items are common, they are not used in day to day situations by the elderly population assessed. Hence, members of both groups also found it difficult to remember items that they have come across but were used only rarely or/and in the early stages of their life. Anju., et al. in 2013 [18] reported similar findings for South Indian DAT population.

Patients with DAT were observed to have an effect of semantic priming. i.e., some of these responses were observed to have a semantic relationship with the stimuli presented. For example: 'fork' as 'ladle' and 'pencil' as 'pen'. This effect could be contributed to the theory that patients with DAT have a difficulty in distinguishing between conceptually similar concepts because similar concepts are difficult to tell apart for patients as they share a large number of same preserved features [19].

There was a positive relationship observed between the presence of cues and the responses obtained in normal aging adults. In the presence of a phonemic cue, the responses were observed to improve in the case of the Group II, i.e. $20 \%$ of the subjects responded correctly. Whereas, there was no such relationship observed in Group I. Melrose., et al. in 2009 [20] reported the neural correlates when a phonemic cue was provided during naming task. They observed that successful naming under this condition was associated with higher metabolic activity in bilateral inferior frontal gyrus (IFG), right superior frontal gyrus (SFG), left temporal and occipital regions.
Similar to the above findings, Cormier, Margison and Fisk in 1991 [21] reported that the performance of Alzheimer's subjects were impaired on all measures of naming and semantic fluency and, when their naming-test errors were analyzed, they showed higher proportions of perceptual errors and failures to respond.

Items 8 (eraser), 14 (pipe) and 16 (elastic) were observed to have had the longest average reaction time compared to other items in the naming task. The reaction time of the items were not related to the accuracy of the answers. The current study revealed that the average latencies for Group I was longer than Group II which was supported by the study done by Passafiume., et al. in 2000 [22] whose results indicated that reading latency is longer in Alzheimer's patients than in normal subjects. Additionally, a study by Nebes and Brady in 1992 [23] revealed that the Absolute Reaction Time (ART) between Alzheimer's patients and normal controls increase as the complexity of the task increases not dependent on the cognitive function tested.

The current study observed paraphasia in very few of the total subjects tested. The paraphasias observed were semantic in nature and not phonological as supported by the study of Bayles and Tolmoeda in 1983 [24] who proposed that phonological paraphasias are rarely produced by DAT patients in confrontational naming tasks.

Also, it was observed that there existed no significant difference in performance between male and female subjects in both the groups.

To conclude, AD patients often demonstrate a progressive decline in performance on tasks that are dependent upon semantic memory, including word finding and picture naming [25]. It is also important to consider the possibility that conscious strategic processing that is needed to access semantic representations may be impaired in the place of actual impaired semantic representations [26]. Melrose., et al. in 2009 [20] reported that naming deficits in $\mathrm{AD}$ may indicate compromise to temporal regions involved in the semantic knowledge network, and frontal regions involved in the controlled retrieval of information from that network.

The verbal fluency task required the rapid generation of words, typically constrained by semantic category. It consisted of 2 sub- 
tasks in it. Subtask I was living things (animals) and II was about man - made objects (supermarket items). The subjects had to say as many names as possible from each category presented to the subject. Each correct response carried a score of one.

D' cruz., et al. in 2013 [13] published the normative score for semantic category verbal fluency in South Indians which was considered as the cutoff normative score in this study. The normative range for the age range of 55 - 80 is $12.58-18.51$ as reported.

\section{Subtask I: Living things (Animals)}

The results indicate that Group I demonstrated a greater impairment in this particular category. Group I obtained a mean value of 7.53 which was relatively lower compared to the 12.4 obtained by Group II. The standard deviation was 3.8303 for Group I and 3.5291 for Group II. Statistical analysis through Independent sample $t$ test revealed that the scores of Group I and Group II were statistically significant with a " $p$ " value of $0.000(p \leq 0.05)$.

Verbal fluency subtask - I (Living things-Animals): Comparison of mean, standard deviation and level of significance between group I and II

Rogers and Freidman in 2008 [27] reported a mean performance in category fluency - living task in Alzheimer's disease as 13.7 which is comparatively lower than the western normative score of 19 . The above results are in coherence with the reports of

\begin{tabular}{|l|c|c|c|c|c|c|}
\hline & $\begin{array}{c}\text { Number of } \\
\text { subjects }\end{array}$ & $\begin{array}{c}\text { Max. } \\
\text { Mean range }\end{array}$ & Mean & $\begin{array}{c}\text { Standard } \\
\text { Deviation }\end{array}$ & SD error & $\begin{array}{c}\text { Level of } \\
\text { significance }\end{array}$ \\
\hline Group I & 30 & $12.58-18.51$ & 7.53 & 3.8303 & 0.6993 & 0.000 \\
\hline Group II & 30 & $12.58-18.51$ & 12.4 & 3.5291 & 0.6443 & 0.000 \\
\hline $\begin{array}{l}\text { (p value } \leq 0.05 \\
\text { indicates significant } \\
\text { level of difference) }\end{array}$ & & & & & & \\
\hline
\end{tabular}

Table 2

Weiner., et al. in 2008 [17]. They observed that the patients with greater cognitive impairment named fewer animals in a minute.

On comparing the verbal fluency of animals in AD patients and MCI group, Brandt and Manning in 2009 [28] reported increased impairment in animal fluency scores than letter fluency. Hodges., et al. in 1990 [29] explained that animal fluency requires the systematic retrieval of hierarchically organized information from semantic memory whereas letter fluency can be successfully performed by relying solely upon phonemic or lexical cues to guide the retrieval process. This distinction in the two tests depending on the structure of semantic knowledge may explain why DAT patients perform poorly on animal fluency compared to letter fluency.

The percentage of repetition was 30\% in Group I indicating more perseverative errors compared to Group II. Group I was observed to produce most common and familiar animals compared to Group II indicating the preservation of information repetitively provided.

\section{Subtask II: Man-made objects (Supermarket items)}

The supermarket fluency task is an indicator of semantic knowledge, and subjects were allotted 60 seconds to name different items that can be found in a supermarket.

Verbal fluency task - II - (Man-made objects-supermarket items): Comparison of mean, standard deviation and level of significance between group I and II

The mean score obtained by Group I and II in the supermarket fluency task were 8.36 and 15.2 respectively indicating a significantly poor performance by Group I. Group II performance was within normal scores. There also existed a statistically significant difference between groups in performance indicating a level of significance of 0.000 ( $\mathrm{p}$ value $\leq 0.05$ ).

Rogers and Freidman in 2008 [27] reported a mean performance in category fluency - non-living task in Alzheimer's disease as 16.7 which is comparatively lower than the western normative 


\begin{tabular}{|l|c|c|c|c|c|c|}
\hline & $\begin{array}{c}\text { Number of } \\
\text { subjects }\end{array}$ & $\begin{array}{c}\text { Max. Mean } \\
\text { score }\end{array}$ & Mean & $\begin{array}{c}\text { Standard } \\
\text { Deviation }\end{array}$ & $\begin{array}{c}\text { SD er- } \\
\text { ror }\end{array}$ & $\begin{array}{c}\text { Level of significance } \\
\text { Group I }\end{array}$ \\
30 & $12.58-18.51$ & 8.36 & 4.246 & 0.775 & 0.000 \\
\hline Group II & 30 & $12.58-18.51$ & 15.2 & 6.299 & 1.150 & 0.000 \\
\hline $\begin{array}{l}\text { (p value } \leq 0.05 \text { indicates significant } \\
\text { level of difference) }\end{array}$ & & & & & & \\
\hline
\end{tabular}

Table 3

score of 19. Their results indicate that the South Indian DAT subjects performed comparatively lower than the western population.

The production of fewer number of words on the supermarket fluency task than the control group was observed and reported by Arroyo-Anlló., et al. in 2011 [31]. They compared patients with AD and control group using the same task. They reported that the total number of correct words produced and the total output (including errors) of each patient group were markedly low in comparison to that of control group.

On specific analysis of the responses, category and exemplars were considered to assess the extent of semantic impairment. A category is defined as a response (e.g. vegetable) which leaves a hypothetical shopper a choice with respect to the particular item someone would buy. An exemplar is defined as a response (e.g. tomato) which leaves a hypothetical shopper with no choice regarding the item someone would buy. Males observed to have produced more categorical response in both the groups whereas females used more exemplars in both the groups. The DAT subjects used more category than exemplars. Comparatively, Group II performed more words per minute than Group I.

As noted by Marczinski and Kertesz, 2006 [32], these qualitative features of the DAT subjects' overall deficits in verbal fluency support the proposal that a bottom-up deterioration of the hierarchical structure of semantic knowledge has occurred. So, DAT subjects show a specific pattern of disruption in the hierarchical structure of semantic knowledge, because the verbal representation of an object is stored with its most general attributes and name at the top of the hierarchy and with its specific features and exemplars at the bottom. While demented patients retain category labels, they generate few exemplars of the categories in fluency task.
The present study also indicates that these changes in semantic memory are present in the early stages of DAT. Most of DAT subjects were characterized by the same qualitative features: i.e. few examples per category and a tendency to an 'over-representation' of category labels. These results are in line with the study done by Arroyo-Anlló., et al. in 2011 [31].

The number of repetition was observed to be same in both the groups. This is in contrast to the results obtained by Arroyo-Anlló., et al. in 2011 [31], who reported that the AD group tends to generate more repetition errors compared to control group.

To conclude the performance on verbal fluency subtasks by male and female in both the groups, males performed comparatively better than females for subtask-I, i.e. animals whereas females performed better than males in subtask-II, i.e. supermarket items. Though there was no significant difference observed statistically.

Functional brain imaging studies by various researchers suggest the category - related semantic memory representations in different cortical regions. Martin and Chao in 2001 [33] reported that the locations of the category- related activations, especially for objects defined primarily by their form related features such as animals, faces and landmarks elicit distinct patterns of neural activity in the ventral occipitotemporal cortex. Similarly, naming man-made objects selectively activates area close to the posterior region of the lateral temporal lobe. This activation of the lateral temporal lobe is not exclusive to naming but also when answering written questions and imagining them.

Yasmeen., et al. in 2017 [34] found that verbal fluency is a sensitive indicator of neurocognitive impairment and is affected and influenced by a number of variables. Semantic fluency is depen- 
dent on only on lexical retrieval speed but also on visualization strategies to support controlled retrieval, skills which may invariably decline with age. In contrast, letter fluency relies heavily on vocabulary knowledge, which itself is dependent on skills acquired and maintained in young to old ages. Semantic memory function is found to be one of the earliest markers of $\mathrm{AD}$, and is indicative of pre Alzheimer's disease neuropathology or mild cognitive impairment $[35,36]$.

Kerns., et al. (1999) [37] puts forth the concept of insufficient storage based on the association between formal thought disorder and loss of semantic information. Some research also indicate difficulty in the retrieval of information from an intact semantic store. This is formed due to considering the fact that impaired fluency may wrongly indicate poor semantic memory [38]. Goldberg., et al. (1998) [39] also reiterates that ability to access semantic storage may be disrupted in the place of actual impairment in semantic storage. This is based on his findings while other measures of language were relatively intact, poor performance on tests sensitive to organization of the semantic system was associated with impaired verbal fluency.

\section{Conclusion}

Overall performance of the individuals with Dementia were poor compared to the normal population. Significant errors issues that could be observed were lexical and semantic errors. There was a positive relationship between phonemic cues and the responses, but a negative relationship between ART and the responses. The perceptual errors and failure to respond might be attributed to the progressive deterioration in the semantic ability of subjects with DAT. In the category fluency task or divergent naming task, the reduced performance of Group I compared to Group II might be blamed on their difficulty for systematic retrieval of hierarchically organised information from semantic memory. The specific characteristic responses observed leads to a postulation about the inability of the subjects to retrieve information from the bottom most part of the semantic memory network.

Results indicate that semantic memory impairments are common in the earliest phases of DAT. Hence, assessment of semantic functions should be central in the speech and language and neuropsychological testing of pre- AD cognitive decline.

It is also valuable to note that Divergent naming and Confrontation naming leads to variable results. This could be due to the visual stimuli present in Confrontation naming compared to the
Auditory stimuli in Divergent naming. Moreover, the widespread distribution of topographical auditory naming sites within the temporal lobe could be a contributing factor. More research on this area could be fruitful in that aspect. Minimal time is required to implement such tests in everyday clinical practice. Confrontation naming is typically impaired more earlier and severely in Alzheimer's Disease and seems to decline in a more linear fashion. In fact, difficulties with naming have even been reported among people with Pre- AD cognitive decline. The contribution of working memory and cognitive flexibility may additionally factor in the performances.

There is a robust relationship observed between semantic processing impairment and everyday functional competencies. Hence, it is important to use different cognitive retraining strategies to improve the semantic processing abilities in subjects with DAT. Thus, we suggest that cognitive retraining of semantic memory network through naming practices would be a useful procedure to follow to improve day to day activities and functional competencies.

\section{Appendix}

Picture naming task

\begin{tabular}{|c|c|c|}
\hline S. No & Stimulus Items & IPA \\
\hline & தப்பாக்கி & /țupa:kki/ \\
\hline & பந்த & /pandu/ \\
\hline & கத்தி & /katisi/ \\
\hline & கப் & $/ \mathrm{k} \Lambda \mathrm{p} /$ \\
\hline & ஊக்கு & /u:kku/ \\
\hline & சுத்தியல் & /sutinjsl/ \\
\hline & ப்ரஷ் & /br^J/ \\
\hline & ரப்பர் & /rubber/ \\
\hline & பூட்டு & /pu:ttu/ \\
\hline & பனெச்ல் & /pensil/ \\
\hline & திருப்புலி & /tiriruppuli/ \\
\hline & சாவி & /sa:vi/ \\
\hline & பபேப்ர்க்ளிப் & /pepırklip/ \\
\hline & கழாய் & /kuzha:i/ \\
\hline & சீப்பு & /si:ppu/ \\
\hline & ரப்பர்பாண்டு & /rıberbænd/ \\
\hline & கரண்ட? & /kar^ndi/ \\
\hline & சலெலேேபேேன்டபே் & /selofentep/ \\
\hline & முள்கரண்டி & /mulkarındi/ \\
\hline & வத்திகுச்சி & /vatikutji/ \\
\hline
\end{tabular}

Table A 


\section{Scores}

Score of 3 - Responds immediately

Score of 2 - Responds with phonemic cues

Score of 1 - Semantic paraphasia

Score of 0 - No response.

Verbal fluency task

Number of animals named in 60 seconds:

Verbatim:

Number of super market items named in 60 seconds:

Verbatim:

Scoring sheet

Picture naming task

\begin{tabular}{|c|c|c|c|c|}
\hline $\begin{array}{l}\text { Item } \\
\text { No. }\end{array}$ & $\begin{array}{l}\text { Latency } \\
\text { (seconds) }\end{array}$ & $\begin{array}{c}\text { Response } \\
\text { Obtained } \\
\text { (Verbatim) }\end{array}$ & Analysis & Score \\
\hline & & & & \\
\hline & & & & \\
\hline & & & & \\
\hline & & & & \\
\hline & & & & \\
\hline & & & & \\
\hline & & & & \\
\hline & & & & \\
\hline & & & & \\
\hline & & & & \\
\hline & & & & \\
\hline & & & & \\
\hline & & & & \\
\hline & & & & \\
\hline & & & & \\
\hline & & & & \\
\hline & & & & \\
\hline & & & & \\
\hline & & & & \\
\hline & & & & \\
\hline
\end{tabular}

Number of stimuli: 20

Number of correct responses: /20

Number of descriptive answers:_/20

Number of incorrect answers: /20

Category fluency task

Number of animals named in 60 seconds:

Verbatim:

Number of super market items named in 60 seconds:

Verbatim:

Score 1 point for every correct response.

No points for repetitions or partial answers.

\section{Bibliography}

1. Eduardo C., et al. "The Neuroanatomical, Neurophysiological and Psychological Basis of Memory: Current Models and Their Origins". Frontiers in Pharmacology (2017).

2. Binder J., et al. "The Neurobiology of Semantic Memory". Trends in Cognitive Sciences 15.11 (2011): 527-536.

3. Mahon BZ and Caramazza A. "A critical look at the embodied cognition hypothesis and a new proposal for grounding conceptual content". Journal of Physiology Paris 102.1-3 (2008): 59-70.

4. Miller KM., et al. "Auditory Responsive Naming versus Visual Confrontation naming in Dementia". The Clinical Neuropsychologist (2009): 103-118.

5. Brandt J., et al. "Auditory Confrontation Naming in Alzheimer's Disease". Clinical Neuropsychology 24.8 (2010): 1326-1338.

6. Tulving E. "Episodic and Semantic Memory" (1972).

7. Hodges JR., et al. "Semantic Memory Impairment in Alzheimer's Disease: failure of Access or Degraded knowledge?". Neuropsychologia 30.4 (1992): 301-314.

8. Thompson-schill L. "Neuroimaging Studies of Semantic Memory: inferring "how" from "where". Neuropsychologia 41.3 (2003): 280-292. 
9. Molloy WD and Lubinski R. "Dementia: Impact and clinical perspectives". In R. Lubinski (Edition.), Dementia and communication (1991): 2-21.

10. Stella Karantzoulis and James E Galvin. "Distinguishing Alzheimer's disease from other major forms of dementia". Expert Review of Neurotherapeutics 11.11 (2011): 1579-1591.

11. Mummery CJ., et al. "A voxel-based morphometry study of semantic dementia: Relationship between temporal lobe atrophy and semantic memory". Annals of Neurology 41.1 (2000): 36-45.

12. Kertesz A. "Western Aphasia Battery" (1982).

13. D'cruz SM., et al. "The effect of aging on verbal fluency in South Indians". IOSR Journal of Dental and Medical Sciences (IOSR-JDMS) 6.3 (2013): 40-42.

14. Hodges JR and Patterson K. "Nonfluent progressive aphasia and semantic dementia: a comparative neuropsychological study". Journal of the International Neuropsychological Society 2 (1996): 511-524.

15. Silagi M., et al. "Naming Ability in patients with Mild to Moderate Alzheimer's Disease: What changes occur with the evolution of the disease?" Clinics 70.6 (2015): 423-428.

16. Kershner HS., et al. "The naming Disorder of Dementia”. Neuropsychologia 22.1 (1984): 23-30.

17. Weiner Neubecker and Bret Hynan. "Language in Alzheimer's Disease”. The Journal of Clinical Psychiatry 69.8 (2008): 12231227.

18. Anju EV., et al. "Semantic Memory in Dementia of Alzheimer's Type". Presented in TAN - ISHA (2013).

19. Martin A. "Semantic knowledge in patients with Alzheimer's disease: evidence for degraded representation". Advances in Psychology 89 (1992): 119-134.

20. Melrose RJ., et al. "The neural correlates of naming and fluency deficits in Alzheimer's disease: an FDG-PET study". International Journal of Geriatric Psychiatry 24.8 (2009): 885-893.

21. Cormier P., et al. "Contribution of perceptual and lexical semantic errors to the naming impairments in Alzheimer's disease". Percept Mot Skills 73.1 (1991): 175-183.
22. Passafiume D., et al. "Reading latency of words and nonwords in Alzheimer's patients". Cortex 36.2 (2000): 293-298.

23. Nebes RD and Brady CB. "Generalized cognitive slowing and severity of dementia in Alzheimer's Disease: Implications for the interpretation of response time data". Journal of Clinical and Experimental Neuropsychology 14.2 (1992): 317-326.

24. Bayles KA and Tomoeda CK. "Confrontational naming impairment in dementia”. Brain Lang 19 (1983): 98-114.

25. Hodges JR and Patterson K. "Is semantic memory consistently impaired early in the course of Alzheimer's disease? Neuroanatomical and diagnostic implications". Neuropsychologia 33 (1995): 441-459.

26. Nebes RD. “Cognitive dysfunction in Alzheimer's disease”. In F. I. Craik and T. A. Salthouse (Editions.), The handbook of aging and cognition. Hillsdale, NJ: Lawrence Erlbaum Association (1992).

27. Rogers SL and Friedman RB. "The Underlying Mechanisms of Semantic Memory Loss in Alzheimer's Disease and Semantic Dementia”. Neuropsychologia 46.1 (2008): 12-21.

28. Brandt J and Manning JK. "Patterns of word list generation in Mild cognitive impairment and Alzheimers disease". Clinical Neuropsychology 23 (2009): 870-879.

29. Hodges JR., et al. "Differential impairment of semantic and episodic memory in Alzheimer's and Huntington's disease: a controlled prospective study". Journal of Neurology, Neurosurgery and Psychology 53 (1990): 1089-1095.

30. Hodges JR., et al. "Semantic dementia: Progressive fluent aphasia with temporal lobe atrophy". Brain 115 (1990): 17831806.

31. Arroyo-Anlló EM., et al. “Verbal fluency in Alzheimer’s disease and Aphasia". Dementia 11.1 (2011): 5-18.

32. Marczinski CA and Kertesz A. "Category and letter fluency in semantic dementia, primary progressive aphasia, and Alzheimer's disease". Brain and Language 97.3 (2006): 258-226.

33. Martin and Chao. "Semantic memory and the brain: structure and processes". Current Opinions in Neurobiology 11 (2001): 194-201. 
34. Yasmeen FS., et al. "Comparison of animal, action and Phonemic fluency in Aphasia”. International Journal of Language and Communication Disorders 53.2 (2017): 370-384.

35. Adlam AL., et al. "Semantic knowledge in mild cognitive impairment and mild Alzheimer's disease". Cortex 42.5 (2006): 675-684.

36. Vogel A., et al. "Semantic memory impairment in the earliest phases of Alzheimer's disease". Dementia and Geriatric Cognitive Disorders 19 (2005): 75-81.

37. Kerns J., et al. "Word Production in Schizophrenia and its relationship to positive symptoms". Psychiatric Research 87.1 (1999): 29-37.

38. Joyce EM., et al. "Verbal Fluency in schizophrenia: relationship with executive function, semantic memory and clinical alogia". Psychological Medicine 26.1 (1996): 39-49.

39. Goldberg TE., et al. "Cognitive Substrates of thought disorder, I: the Semantic system". The American Journal of Psychiatry 155.12 (1998): 1671-1676.

40. Huff FJ., et al. "Semantic Impairment and Anomia in Alzheimer's disease". Brain and Language 28.2 (1986): 235-249.

41. Hodges JR and K Patterson. "Nonfluent progressive aphasia and Semantic dementia: a comparative neuropsychological study". Journal of the International Neuropsychological Society: JINS 2.6 (1996): 511-524.

\section{Volume 3 Issue 9 September 2021}

(C) All rights are reserved by Deepthi Vivek V and Amirtha Varshini MJ. 